\title{
Bernard ALLEMANDOU, Jean-Jacques LE PENNEC, Les orphelins, enfants de la Patrie, Pessac, Maison des Sciences de l'Homme d'Aquitaine, 2002, 271 p.
}

Pascale Quincy-Lefebvre

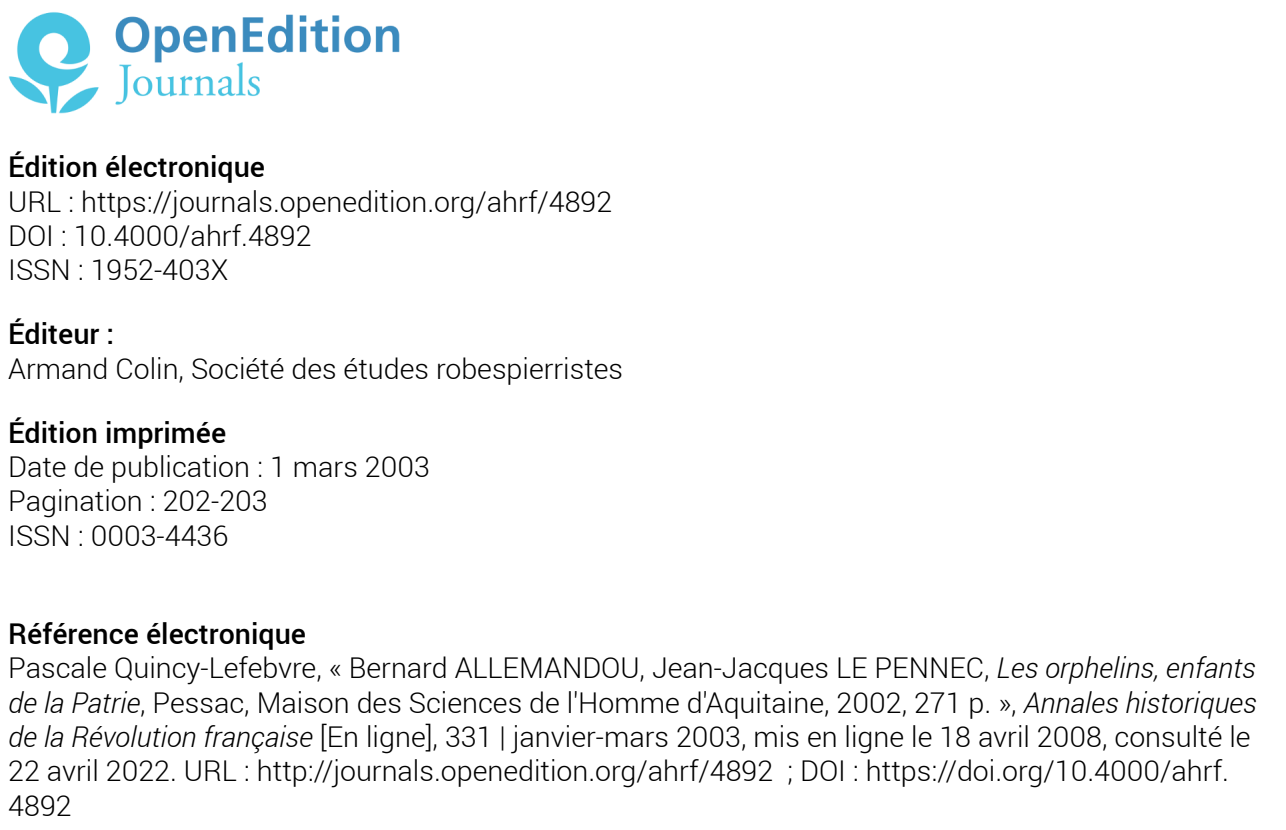

Ce document a été généré automatiquement le 22 avril 2022.

Tous droits réservés 


\title{
Bernard ALLEMANDOU, Jean- Jacques LE PENNEC, Les orphelins, enfants de la Patrie, Pessac, Maison des Sciences de l'Homme d'Aquitaine, 2002, 271 p.
}

\author{
Pascale Quincy-Lefebvre
}

1 L'ouvrage peut surprendre l'historien de formation. Rédigé par deux médecins, il poursuit une aventure commencée en 1991 avec la sortie d'un premier volume intitulé La naissance de l'aide sociale à l'enfance à Bordeaux sous l'Ancien Régime. À terme, le projet de cette histoire générale de l'aide sociale à l'enfance à Bordeaux devrait mener les auteurs jusqu'à l'époque contemporaine. Un travail rare et une ténacité exemplaire.

2 Insérée dans un programme de recherche sur «La municipalisation des politiques de Santé » placé sous la responsabilité de Jean-Claude Guyot, professeur de sociologie à Bordeaux 2, l'étude a l'ambition d'être exploitable par les travailleurs sociaux ou les élèves en formation du secteur concerné. Empruntant ses concepts à la sociologie des organisations et mobilisant des grilles de lecture empruntées au travail social, l'équipe explore le temps, soucieuse qu'elle est, au milieu de quelques digressions ou curiosités, de rendre compte des cheminements aboutissant à l'actuelle structuration de «l'enfance en marge».

3 S'appuyant sur un certain nombre de lectures de référence (avec quelques oublis qu'on peut regretter), les auteurs ont mis à jour une documentation précieuse qui vient confirmer mais également enrichir notre connaissance du sujet.

4 La période révolutionnaire, comme crise majeure de notre histoire, ne pouvait que passionner Bernard Allemandou et Jean-Jacques Le Pennec dans une enquête portant sur la manière dont la société compose une réponse sociale au problème posé par l'enfance en marge. La question qu'ils posent est commune mais centrale: la Révolution a-t-elle changé le sort réservé aux enfants trouvés et les institutions les 
prenant en charge ? Le mérite est d'essayer de mesurer à l'échelon local ce qui fait continuité et rupture avec l'Ancien Régime.

5 L'histoire institutionnelle est menée de façon classique, qui insiste sur la désorganisation née des nouveaux principes administratifs et des désordres politiques. Les auteurs ont réalisé un réel travail sur archives à partir de fonds concernant l'hôpital de la Manufacture des enfants transformé en hospice des enfants de la Patrie. Avec difficulté et un absentéisme endémique des administrateurs de l'hospice, c'est la naissance d'une gestion de type administratif, où toutes les tâches sont répertoriées, qui décide du sort de l'institution. La situation s'éclaircit avec la loi du 16 vendémiaire an $\mathrm{V}$ qui place l'hospice sous l'autorité de la commission administrative des hospices de la ville. Au plan institutionnel, les auteurs nous confirment l'échec de la centralisation révolutionnaire justifiant que, à la fin du Directoire, on en revienne au principe d'une gestion municipale et d'un financement local par le rétablissement de l'octroi.

6 L'imbroglio administratif, les difficultés financières qui, par exemple, obligent le trésorier à faire des avances sur ses fonds propres ont certainement joué au profit des sœurs, élément stable jusqu'en décembre 1794. Avant cette date et bien après le décret du 18 août 1792 qui supprime les congrégations, les administrateurs ne peuvent ou ne veulent régler le problème des sœurs au sein de l'hospice.

7 Les auteurs ne s'arrêtent pas sur un simple constat d'échec des révolutionnaires dans l'organisation de «l'aide sociale » à l'enfance. Ils tentent d'identifier « une révolution culturelle » dans la représentation de l'enfance en marge, ici l'enfance exposée. Dans un monde en crise, ils font émerger de leurs sources normatives un nouvel idéal et ils s'interrogent pour savoir si l'idéal a transformé le réel de l'institution mais également des familles.

8 La Révolution fait accéder les enfants abandonnés à un statut fixé par la loi. Le préjugé de bâtardise est refoulé. Objets d'une nouvelle considération, ils deviennent les orphelins de la patrie, susceptibles d'être adoptés. Expression de la Nation, cette décision reflète une histoire particulière accrochée aux événements. Elle en dit long sur l'idée d'État. Elle appartient également à une histoire plus profonde d'une modernisation des représentations de l'autre et plus particulièrement d'un sentiment d'enfance en pleine refondation.

9 Les transformations sur le réel sont difficiles à saisir. À côté de quelques déclarations de principe des administrateurs, la mortalité terrible des enfants relativise l'emprise de cette considération. Cette situation ne désarçonne pas les auteurs qui traquent les signes de cette révolution culturelle dans les pratiques parentales et dans le rôle de l'institution. Observant une croissance relative des abandons à Bordeaux, ils relèvent la part des enfants légitimes dans la population abandonnée et, à travers les opérations de restitution aux familles, ils identifient un nouvel usage social de l'institution, qui transformerait l'établissement de lieu de renfermement structuré par la honte en un lieu pouvant davantage être conçu comme un établissement de placement temporaire.

10 Les signes sont intéressants même s'ils demandent à être relativisés. Ils mériteraient d'être appuyés par d'autres exemples et d'autres études. Le prochain volet est attendu. 